\title{
High-Precision Forming Process Based on Analysis of Selective Laser Sintering Hybrid Milling
}

\author{
Trung Nghiep NGUYEN ${ }^{* *}$, Hideki AOYAMA ${ }^{* * *}$ and Ichiro ARAIE ${ }^{* * * *}$
}

\begin{abstract}
Selective laser sintering hybrid milling (SHM) is an advanced manufacturing technology that combines the advantages of additive manufacturing and traditional machining. This technology is used to fabricate extremely complicated and high-precision products. The key factor of SHM is the milling process that is performed during the depositing process. However, the thermal shrinkage during the cooling process when depositing new layers on the ones that were machined is a fundamental problem because it creates deformation errors which significantly reduce product accuracy. In this study, the thermal deformation error in SHM was investigated using finite element analysis (FEA) and confirmed experimentally. The mechanism involved in the shrinkage in SHM was studied using three shrinkage rules. Moreover, a compensation technique was proposed with the aim of reducing the thermal deformation error. The results proved that compensation could significantly reduce the deformation error in SHM.
\end{abstract}

Key words: SLS hybrid milling, thermal deformation error, finite element analysis, compensation

\section{Introduction}

Additive manufacturing (AM) has numerous advantages owing to the possibility of fabricating extremely complicated products that are impossible to create by traditional machining ${ }^{1}$. However, low accuracy and poor surface quality are the fundamental limitations of this technology 2). To solve this problem, new versions of three-dimensional (3D) printing machines, combined with high speed milling have been developed ${ }^{3}$. This technology is based on the concept of additive/subtractive hybrid manufacturing (ASHM) ${ }^{4}$. According to ASHM, milling is conducted alternatively at the same time, not after the forming process. Unfortunately, forming new layers on the finished ones creates deformation errors owing to the shrinkage phenomenon, which significantly reduces the integrity of product. Therefore, it is extremely important to find solution to thermal deformation error for developing the ASHM technology in the current competitive machine tools market.

Additive/subtractive hybrid manufacturing provides advantages of both AM and conventional machining. This technology allows fabricating complicated product with high precision and high surface quality. Several methods of deposition of metals such as 3D welding ${ }^{5)}$, laser deposition of metals ${ }^{\text {9 }}$, laser wire deposition ${ }^{7}$, selective laser cladding (SLC) ${ }^{8)}$, and selective laser sintering (SLS) ${ }^{4}$ could be used in ASHM. The technology that combines both SLS and milling; is known as SLS hybrid milling (SHM); was first proposed by the researchers of Panasonic ${ }^{7,29}$. Recently, machines based on this technology have been released into the market to advertise accuracy of $\pm 0.01 \mathrm{~mm}{ }^{8}$. The process of SHM involves a repetition of deposition of metal using SLS and milling process ${ }^{4}$. First,

\footnotetext{
" Received on October 7, 2019

Accepted on March 31, 2020

* Student member, Keio University (3-14-1 Hiyoshi, Kohoku-ku, Yokohama, Kanagawa 223-8522, JAPAN)

*** Full member, Keio University (3-14-1 Hiyoshi, Kohoku-ku, Yokohama, Kanagawa 223-8522, JAPAN)

**** Full member, Sodick Co., Ltd (Ka-11, Miya-Machi, Kaga-shi, Ishikawa 922-0595, JAPAN)
}

approximately 10 to 25 layers of metal are deposited on the base plate by SLS. Next, this block of layers is machined by milling on the same work station. Subsequently, the same number of layers is continuously deposited and machined on the first block. This process is continuously repeated until all layers of the product are formed.

The SHM technique was developed to solve the problems of low accuracy and poor surface quality of the metal powder bed fusion. Moreover, this technology allows even the inner surfaces of products to be machined. One of the applications of SHM is fabricating injection molds with optimized internal cooling channels. Several products of this application are displayed on the website of Sodick Co., Ltd ${ }^{8}$. However, thermal deformation error reduces the accuracy of SHM. As mentioned above, in SHM, new layers of metal are continuously deposited on the layers that were precision machined earlier. Shrinking of the new layers during their cooling causes deformation of the previous layers. Thermal deformation error, therefore, appears on the surfaces of finished products. Since the thermal deformation error could significantly affect the accuracy of SHM, we aimed to find a technique to reduce that kind of error.

Although understanding of thermal deformation error in SHM is important, literature reports no study on this aspect. SHM has been developed from SLS technologies; however, studies on the accuracy and surface quality in SLS are scarce. This is attributed to the fact that product of SLS are usually not used directly but continuously finished by computer numerical controlled CNC milling owing to low accuracy and poor surface quality. Another reason is that SHM machine is currently not popular although some companies have already released their SHM-derived products into the market. Current research in the field of metal-based AM technology focuses on mechanical properties ${ }^{4), 9)(2)}$, microstructure 2 , 4), 9), 10), 11), and residual stress ${ }^{12)-20}$. Studies related to thermal deformation, have only investigated for geometrical error in SLS ${ }^{10}$, 19), but not the thermal deformation error.

The target of the present research is investigating thermal deformation error in SHM and providing an efficient method to increase the accuracy. In the present study, a model based on Finite Element Analysis (FEA) method was developed to simulated the forming process of SHM. 
Dimension of the testing sample after deformation owing to shrinkage is determined by simulation, consequently, confirmed with experiment. Based on deformation error achieved from the simulation, the compensation approach was developed. The second simulation and experiment were conducted to confirm the effectiveness of proposed compensation strategy. The result proved that compensation technique which used deformation error achieved from FEA simulation could significantly improve the accuracy of SHM. On the next sections of this paper, first, the FEA model used to calculate thermal deformation error is explained. Next, results of simulation and experiment in both cases with and without compensation are reported in detail. On the discussion section, proposed FEA model is evaluated, and the effectiveness of compensation method is discussed.

\section{Methods}

In the present research, simulation by FEA was selected as the main study method because performing experiment with SHM machines is costly and time consuming. Moreover, as mentioned above, SHM machines are not popular yet; therefore, performing experiment with SHM machines is considerably difficult. Thus, FEA is expected to reduce the time and expenditure in collecting data. The FEA model needs to satisfy three requirements. First, it must be able to logically explain the mechanism of thermal deformation in SHM. Second, the gap between the result of simulation and experiment must be smaller than the requirement of accuracy in SHM. In the current commercial SHM machines, the requirement accuracy is $0.01 \mathrm{~mm}$. Finally, the time of simulation must be as short as possible.

Currently, there are no studies that report FEA models for SHM.

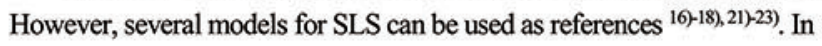
most of these models, the scanning process is simulated by moving a heat source on a 3D model. While there are usually hundreds of scanning lines in one layer, time for heat simulation of a single layer requires several hours. This explains why researchers always used models with small dimension such as $1 \times 5 \times 1 \mathrm{~mm}^{18)}$, or $7 \times 4 \times 1 \mathrm{~mm}^{22}$. Moreover, only one or a few layers get simulated, whereas there exist usually hundreds to thousands of layers in one sample of SLS. Thus, these FEA models are only suitable for studying heat distribution, welding pond, and microstructure or residual stress. For calculating the thermal deformation error of an SHM sample, all layers must be simulated. Therefore, using a similar FEA model as in the current research will involve several months, even a year for only one simulation. Hence, a simpler FEA model was proposed for SHM and obtained expected results.

\subsection{Simulation process}

In the present research, the FEA model was developed based on a real forming condition in the OPM250L machine. Testing sample model is shown in Fig. 1 (a). Material for the base plate was S50C without hardening, and material of metal powder was Maraging Steel (Table 2) ${ }^{24)}$, 25). The length of model was $60 \mathrm{~mm}$, and the width of model was $10 \mathrm{~mm}$. The two walls on the $\mathrm{x}$-axis of this test sample were named left and right Fig. 1 (b). The objective of this model was to calculate the deformation along the $\mathrm{x}$-axis on the left and right walls. The height of the sample was $20 \mathrm{~mm}$, whereas the thickness of one layer was $0.04 \mathrm{~mm}$. Therefore, this test sample had 500 layers deposited by SLS. These 500 layers were divided by 20 blocks, and each block had 25 layers (Fig. 2). Thickness of (a) Dimensions of sample

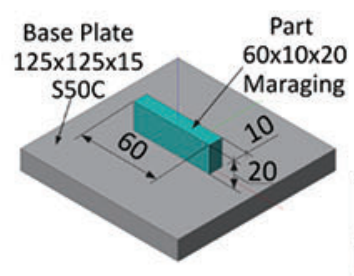

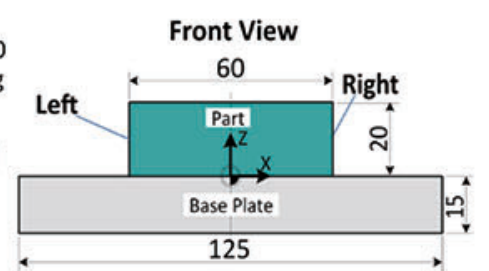

(b) Front view
Fig. 1 Testing sample

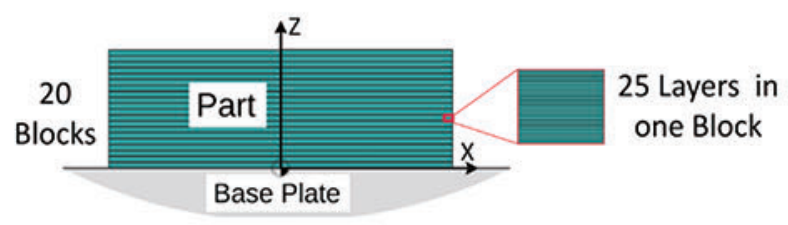

Fig. 2 Number of layers in one block

one block was $1 \mathrm{~mm}$, and the forming process had 22 steps.

Step 1:

Step 1 is shown in Fig. 3. First, 25 layers were alternatively deposited on the top of base plate by SLS (Fig. 3 (a)), and clubbed under the name "block 1". Approximately $0.2 \mathrm{~mm}$ of extra material was added to the subsequent milling process. Next, in the milling process of step 1, block 1 was rough machined (Fig. 3 (b)). An area of $0.035 \mathrm{~mm}$ was left for finishing milling to be performed in the next step.

Step 2:

Step 2 is shown in Fig. 4. First, 25 new layers were continuously to be deposited on the top of block 1 (Fig. 4 (a)). These constituted the block 2. Similar to bock 1 , block 2 was rough machined by milling and $0.035 \mathrm{~mm}$ area was left for finishing milling (Fig. 4 (b)). After rough milling of block 2, block 1 was finish machined (Fig. 4 (c)). The above-mentioned technique that performed rough and finishing milling in two different steps is known as step machining process (Patent No. 3687672) ${ }^{29}$. There are two differences between simulation model and the real milling process. The first difference is that in the real milling process, ball milling tool was used for rough milling, therefore it was impossible to machine the $90^{\circ}$ corner between the wall block 1 and the top face of the base. However, in simulation we assumed that corner was perfectly machined as shown in Fig. 3 (b). The second difference is that in our simulation, the finishing milling was assumed to be performed exactly only on one block at the corresponding step (Fig. 4 (c)). In the real milling process, a small part of the above block was also machined (Fig. 5). This technique ensured that the whole block is finished machined. In simulation, we simplified the milling process as explained above to reduce the simulation time. The final results of the research confirmed that this modification insignificantly affected to the simulation results.

Step 3, 4,..., 20:

Next, steps $3,4, \ldots, 20$ were conducted similar to step 2 . After step 20 , 20 blocks were formed and the height of the sample reached $20 \mathrm{~mm}$, which was the target dimension.

Step 21:

Although, the height of the sample had already reached the target dimension in step 20,10 more layers were continuously deposited on the top of block 20. These extra layers ensured that the height of the sample was above the requirement. The extended height was to be machined later to create the finishing top face. After depositing the extra layers, the top and the walls of block 20 were finished in the milling process of step 21 . 


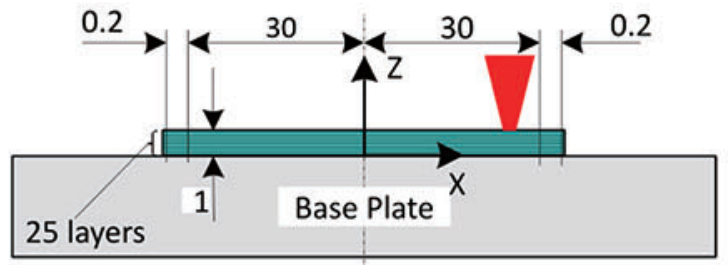

(a) Depositing by SLS

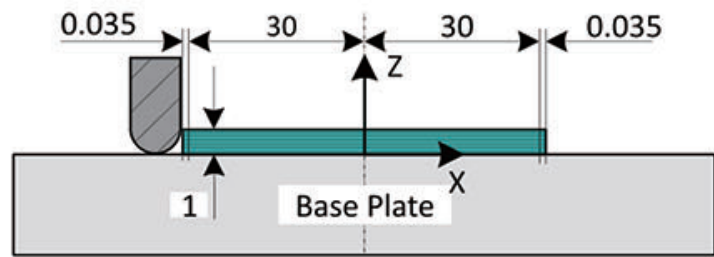

(b) Rough cutting by milling

Fig. 3 Step 1 of forming process

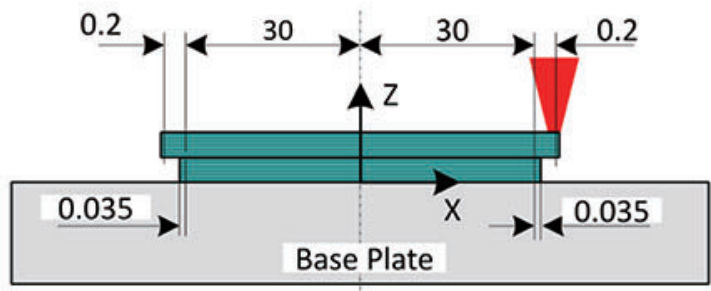

(a) Depositing by SLS

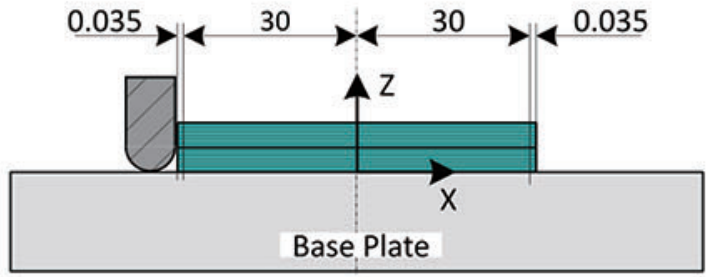

(b) Rough cutting for Block 2

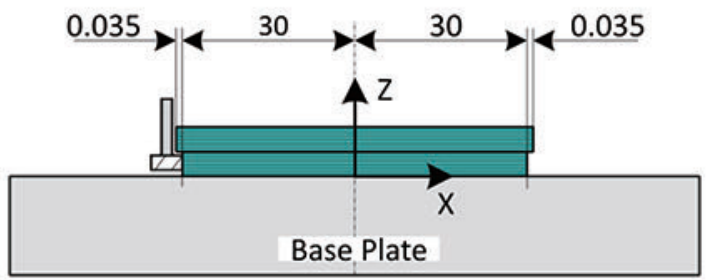

(c) Finishing cutting for Block 1

Fig. 4 Step 2 of forming process

Step 22 (Unclamping):

After finishing forming the part, it was unclamped and remove from the workstation. Unclamping was considered one step although it was simple step because this step also created more deformation in the part.

In the present research, Code Aster was used for building the FEA model. To reduce the simulation time, a two-dimensional (2D) FEA model, instead of 3D model, was used to calculate thermal deformation. In this 2D model, "plane stress" analysis was used because the width of the part (10 mm) is small compared with the length of the part $(60 \mathrm{~mm})$. As mentioned above, the real forming process had 22 steps; the simulation, therefore, also had 22 sub-simulations. One sub-simulation calculated the depositing process of all layers in one block. Each sub-simulation used a specific meshing model designed to simulate the forming process of 25 layers continuously. Figs. 6 and 7 show the model before and after meshing of step 5. In one sub-simulation, a coupled thermal mechanical

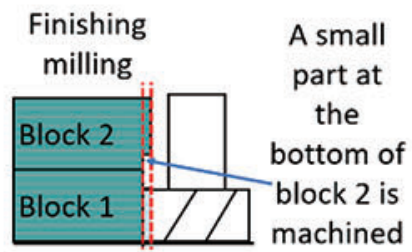

Fig. 5 Finishing milling of step machining process in real

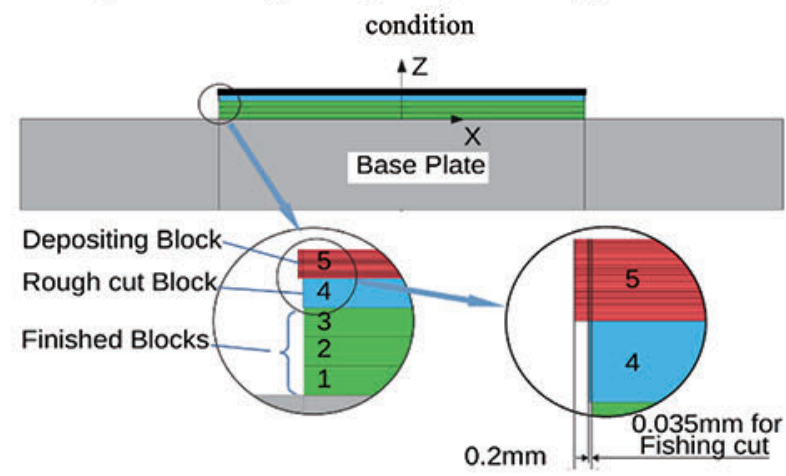

Fig. 6 Simulation model for the forming process of Block 5 before meshing

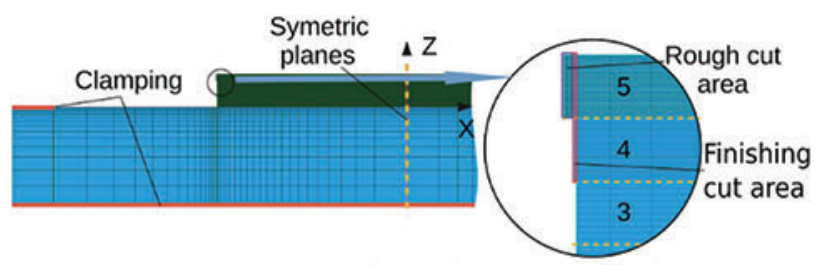

Fig. 7 Simulation model for the forming process of Block 5 after meshing

simulation was used to simulate the SLS process. After one sub-simulation, a result file was exported that was then imported to the next sub-simulation as the initial condition.

As illustrated in Fig. 7, the present research used quadrangle elements. The elements that contained the points of left and right walls had the smallest sizes. Within these elements, the elements on the finished machined blocks had the size of $0.6 \times 0.1 \mathrm{~mm}$, the elements in the rough machined block but not in cutting area had the size of $0.6 \times 0.04 \mathrm{~mm}$, the elements in the rough machined block and in cutting area had the size of $0.035 \times 0.04 \mathrm{~mm}$, the elements in the new layers but not in cutting area had the size of $0.6 \times 0.0133 \mathrm{~mm}$, and the elements in the new layers and in cutting area had the size of $0.035 \times 0.0133 \mathrm{~mm}$. The elements which were farther to the left and right wall had the larger sizes. This design of the mesh guaranteed that the results at the position we wanted to investigate (left and right wall) were precise and accurate while simulation time could be minimized.

\subsection{Thermal simulation}

As mentioned above, deformation error is owing to shrinkage; thus, we predicted that it is created mostly in the cooling process when material is shrinking. Therefore, in the present FEA model, we simplified the scanning stage, and only conducted the simulation for cooling stage. From the real forming process, it was estimated that cooling time of one layer was $30 \mathrm{~s}$. At the beginning of cooling process of a particular layer, the temperature of that layer was set $1500^{\circ} \mathrm{C}$ (the forming temperature). The forming temperature of that layer was set uniformly; that means all of the nodes in the layer were set $1500^{\circ} \mathrm{C}$. 
Table 1 Sampling time of FEA model

\begin{tabular}{ccc}
\hline \multicolumn{2}{c}{ Cooling process } & $\begin{array}{c}\text { Sampling time of the } \\
\text { FEA model }\end{array}$ \\
\hline From & To & $0.000001 \mathrm{~s}$ \\
$0.00001 \mathrm{~s}$ & $0.00001 \mathrm{~s}$ & $0.00001 \mathrm{~s}$ \\
$0.0001 \mathrm{~s}$ & $0.0001 \mathrm{~s}$ & $0.0001 \mathrm{~s}$ \\
$0.001 \mathrm{~s}$ & $0.001 \mathrm{~s}$ & $0.001 \mathrm{~s}$ \\
$0.01 \mathrm{~s}$ & $0.01 \mathrm{~s}$ & $0.01 \mathrm{~s}$ \\
$0.1 \mathrm{~s}$ & $0.1 \mathrm{~s}$ & $0.1 \mathrm{~s}$ \\
$1 \mathrm{~s}$ & $1 \mathrm{~s}$ & $0.5 \mathrm{~s}$ \\
$5 \mathrm{~s}$ & $5 \mathrm{~s}$ & $1 \mathrm{~s}$ \\
\hline
\end{tabular}

Table 2 Material properties

\begin{tabular}{lll}
\hline Material properties & Maraging steel & S50C \\
\hline$\rho C\left(\mathrm{~J} / \mathrm{m}^{3} \mathrm{~K}\right)$ & 2686700 & 3841600 \\
$k(\mathrm{~W} / \mathrm{mK})$ & 19.7 & 50.2 \\
$\alpha\left(\mathrm{K}^{-1}\right)$ & $1.009 \mathrm{E}^{-5}$ & $1.1 \mathrm{E}^{-5}$ \\
$\varepsilon$ & 0.3 & 0.3 \\
$E(\mathrm{GPa})$ & Fig. 9 (a) & 208 \\
$E_{t}(\mathrm{MPa})$ & Fig. 9 (b) & 100 \\
$S_{y}(\mathrm{MPa})$ & Fig. $9(\mathrm{c})$ & 375 \\
\hline
\end{tabular}

The forming temperature was given for $10^{-6} \mathrm{~s}$ (equal with sampling time) (Table 1). The assumption of forming temperature was based on experience and reference of melting temperature of maraging steel $\left(1413^{\circ}\right.$ C) ${ }^{27}$. The final results confirmed that this assumption was appropriate with experiment. At the beginning of cooling process, speed of heat transferring was extremely fast owing to the large gap of temperature between the new formed layer and bulk of material below. The speed of heat transferring reduced significantly after a few seconds. To guarantee both accuracy of calculation and time saving, sampling time of FEA model was extremely small at the beginning, and was increased after that. The detail of sampling time is given in Table 1.

Thermal simulation was based on the heat transferring Eq. (1) given below:

$$
\rho C \frac{\partial T}{\partial t}=\frac{\partial}{\partial x}\left(k \frac{\partial T}{\partial x}\right)+\frac{\partial}{\partial z}\left(k \frac{\partial T}{\partial z}\right)+Q
$$

where $\rho$ is the density of material $\left(\mathrm{kg} / \mathrm{m}^{3}\right) ; C$ is the specific heat capacity $(\mathrm{J} / \mathrm{kgK}) ; T$ is the temperature at calculating point $(\mathrm{K}) ; t$ is time (s); $k$ is thermal conductivity $(\mathrm{W} / \mathrm{mK})$, and $Q$ is the heat loss at the opened surface of the part and base plate. $\rho C$ and $k$ were assumed unchanged and are given in Table 2; $Q$ is given by Eq. (2):

$$
Q=h A\left(T_{E x t}-T\right)
$$

where $h$ is heat transfer coefficient $\left(\mathrm{W} / \mathrm{m}^{2} \mathrm{~K}\right) ; T_{E x t}$ is room temperature. In the present study, $h$ and $T_{E x t}$ were assumed unchanged and are alternatively equal to $100 \mathrm{~W} / \mathrm{m}^{2} \mathrm{~K}$ and $293 \mathrm{~K}\left(20^{\circ} \mathrm{C}\right)$. The initial temperature of base plate was also assumed to be $20^{\circ} \mathrm{C}$.

\subsection{Mechanical simulation}

Mechanical simulation was always performed after thermal

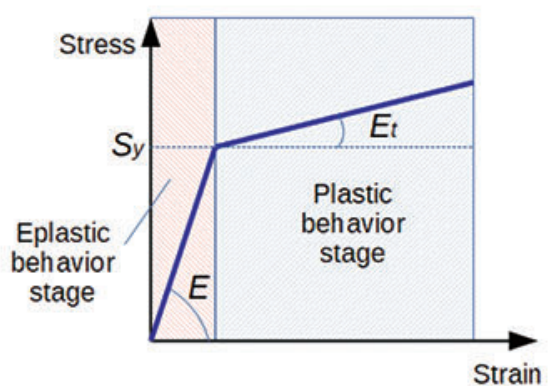

Fig. 8 Elastoplastic behavior of material ${ }^{28}$ )

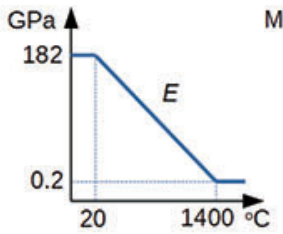

(a) Young's modulus

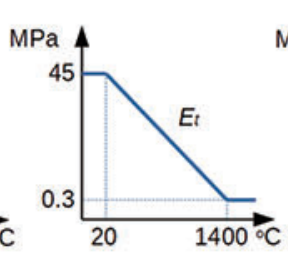

(b) Tangent

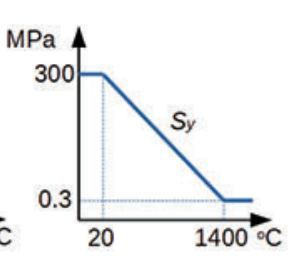

(c) Yield point
Fig. 9 Properties of maraging steel

simulation. Results of thermal simulation were the input data to calculate shrinkage in mechanical simulation. During the cooling process, it was predicted that both plastic and elastic deformation occurred. This behavior of material is called elastoplastic behavior. Therefore, in the present study, the elastoplastic behavior of Code Aster was used. The specific material properties that define elastoplastic behavior of Code Aster include Young's modulus $(E)$, elastic limit or yield point $\left(S_{y}\right)$, and tangent modulus $\left(E_{t}\right)$. The stress strain curve is shown in Fig. 8 that explains the meaning of these parameters in detail ${ }^{28}$ ). In this FEA model, the three parameters of maraging steel were defined as the function of temperature (Fig. 9), and these values for S50C were assumed constant and given in Table $2{ }^{24)}$ 25). Regarding the data in Fig. 9, in fact, references only provided the properties of maraging steel at the normal condition $\left(20^{\circ} \mathrm{C}\right.$ to $80^{\circ} \mathrm{C}$ ) while the simulation needed the properties up to $1500^{\circ} \mathrm{C}$. The missing data could not be found in any reference. Therefore, we needed to predicted the missing parameters by experience. Based on the reference of maraging steel melting temperature, we assumed that at $1400^{\circ} \mathrm{C}$, the material begins to melt. The mechanical properties of material at higher $1400^{\circ} \mathrm{C}$ was assumed to be extremely small compared with that ones at normal condition. And the relationship between missing parameters and temperature was assumed to be linear as shown in Fig. 9. The assumed parameters were tested before applying for the present research. The final results of the present research also confirmed that the assumption above is appropriate. Two more important parameters used to calculate shrinkage are thermal expansion coefficient $(\alpha)$ and Poisson's ratio $(\varepsilon)$. These parameters are given in Table 2. For simulation of the clamping condition, the bottom edge and two elements at corners of the base plate were fixed in both directions $X$ and $Z$ (Fig. 7). In the present research, we ignored the phase transformation from liquid to solid of metal. It was predicted that shrinkage at the stage of liquid of metal does not affect significantly to deformation error. The final results showed that the prediction was appropriate.

\subsection{Simulation of continuous layers in one block (using elements birth and death)}

As mentioned above, one block had 25 layers, and all of these were simulated in one sub-simulation with only one mesh 


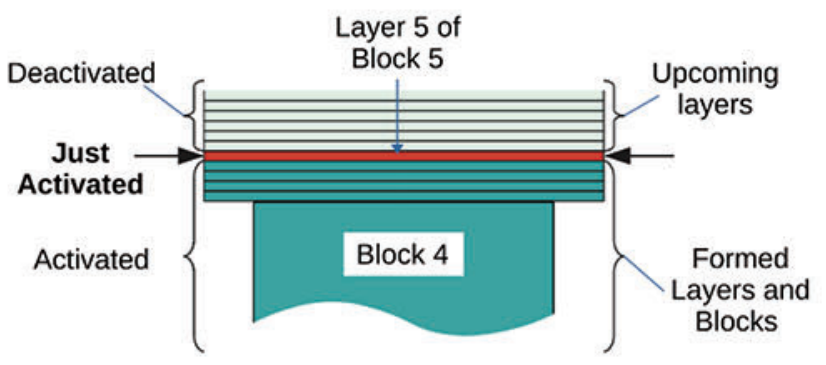

Fig. 10 Activating layer 5 of block 5

model. To achieve this, the elements birth and death technique was used. This technique is illustrated in Fig. 10 with the depositing process of layer 5 of block 5 . In the actual depositing process of layers, it is obviously that when layer 5 of block 5 is deposited, layers 6 to 25 of this block are not existed yet. However, the mesh model used to simulate the deposition process of all 25 layers must have all of nodes of these 25 layers. But, when layer 5 of block 5 is simulated, the nodes of layers 6 to 25 must be ignored in the calculation. The nodes that are ignored and do not considered at this step of the simulation is termed deactivated nodes (death nodes). In contrast, the nodes joint in the calculation is termed activated nodes. When a new layer is simulated, the first thing needs to do is convert the stage of the nodes in this layer from deactivated to activated. Therefore, when the simulation for layer 5 began, all elements in layer 5 got activated, and the forming temperature was set to $1500^{\circ} \mathrm{C}$ (as explained in section 2.2), and the cooling process began as explained in section 2.2. At this point, all elements below layer 5 are already activated; however, the above elements must be deactivated. Therefore, they are not affected during the cooling process of layer 5 . After $30 \mathrm{~s}$, the cooling process of layer 5 is finished, and layer 6 is continuously activated. This process is repeated until all layers of block 5 are finished.

\subsection{Simulation of milling process}

Considering milling effect during a special requirement of our FEA model. Because the other models for SLS do not have milling process, we proposed a new technique to simulate milling process in this study. First, we assumed that error of milling process to be zero. For simulating the cutting process, the FEA model was specifically designed with separating milling areas for both rough milling and finishing milling (Fig. 6 and 7). After simulating the depositing process of all 25 layers in a block and before continuing the simulation for the next block, the rough milling area and finishing milling area of corresponding blocks were removed. The deformation on the just milled blocks was reset to $0 \mathrm{~mm}$. The model now was considered as just machined and ready for the deposition of next layers. The milling simulation of step 5 is illustrated in Fig. 11. After simulating the depositing process of 25 layers of block 5 , we got the deformation result as illustrated in Fig. 11 (a). After that, we remove the elements in the rough cutting area of block 5 and finishing cutting area of block 4 . Then deformation of the nodes in block 4 and block 5 is reset to be zero as illustrated in Fig. 11 (b). Consequently, this result will be imported to the next step and used to set as the initial condition.

In the above example of milling simulation, before milling, there is strain in block 4 and block 5 , which is called strain before milling.

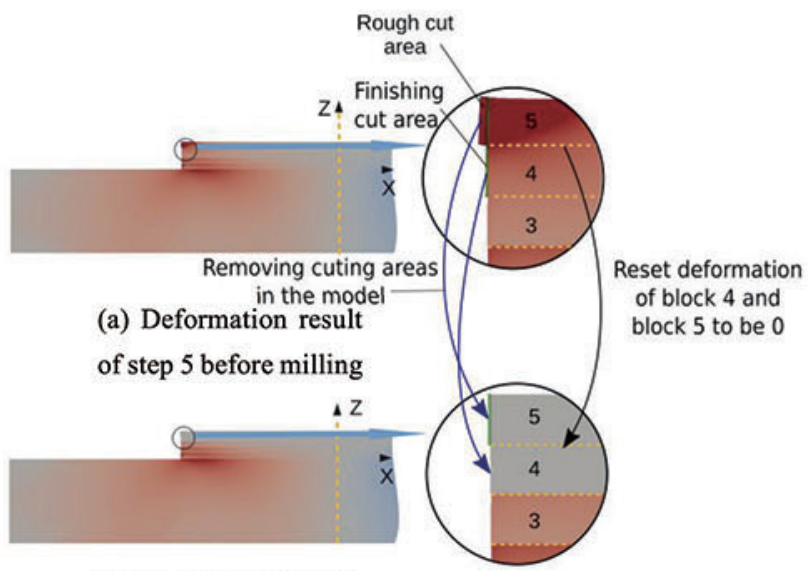

(b) Deformation result

of step 5 after milling

Fig. 11 Simulation of milling process in step 5

Top view (Odd Number Layers)

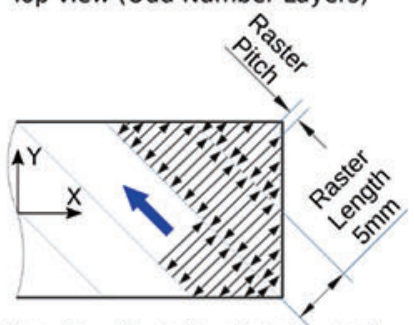

Top view (Even Number Layers)

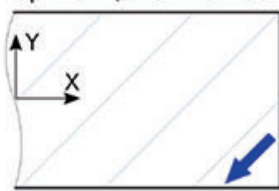

(a) Scanning pattern

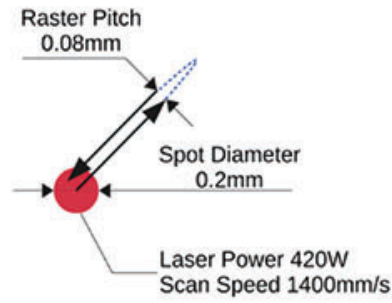

(b) Laser power, scans, spot diameter, raster pitch
Fig. 12 Scanning conditions

However, in SHM technology, the strain before milling does not contribute to the deformation error. Therefore, the simulation only needs to calculate the strain after finish-milling. That explains why in the simulation, the strain before milling is reset to be zero.

\subsection{Forming conditions in experiment}

In the present study, experiments were performed to confirm and evaluate the results of simulation. The forming process of the real samples was completely similar to forming process of simulation. The parameters not considered in simulation are given below. First, the other SLS process parameters, including scanning pattern, raster pitch, raster length, laser power, scanning speed, and laser spot diameter, are explained and given in Fig. 12. In the real milling process, there was one point which differs with simulation. Instead, there was only one step of rough milling in simulation; in the real process, there were two continuous steps: rough milling and semi-finishing milling. This modification helped reduce the complication of the FEA model. Since the effect of milling error was assumed to be insignificant, this modification did not affect to simulation results. Milling conditions are given in detail in Table 3. These milling conditions were tested to confirm that the milling error was not significant, when compared with the thermal deformation error.

\section{Simulation and experiment results}




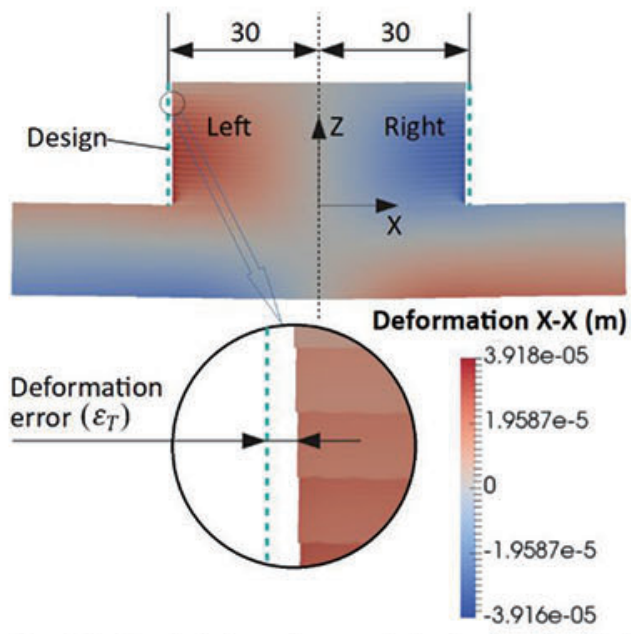

Fig. 13 Calculated deformation result in the first simulation

Table 3 Milling conditions

\begin{tabular}{lccc}
\hline & Rough milling & $\begin{array}{c}\text { Semi-finishing } \\
\text { milling }\end{array}$ & $\begin{array}{c}\text { Finishing } \\
\text { milling }\end{array}$ \\
\hline Tool & Ball end-mill & Ball end-mill & T end-mill \\
\hline Tool radius & $1 \mathrm{~mm}$ & $1 \mathrm{~mm}$ & $0.5 \mathrm{~mm}$ \\
\hline Revolution (rpm) & 17000 & 17000 & 25000 \\
\hline $\begin{array}{l}\text { Feed rate } \\
\text { (mm/min) }\end{array}$ & 1200 & 1200 & 1200 \\
\hline $\begin{array}{l}\text { Radius depth of } \\
\text { cut (mm) }\end{array}$ & $\approx 0.1$ & 0.065 & 0.035 \\
\hline Z-pitch (mm) & 0.08 & 0.08 & 0.05 \\
\hline XY-pitch (mm) & 0.08 & 0.08 & 0.05 \\
\hline
\end{tabular}

In the present study, two simulations were conducted: without compensation and with compensation. Forming without compensation means that the designed dimension of sample was used as the setting value for finishing milling. Forming with compensation means that the setting value for finishing milling was the added deformation error, which was calculated using the first simulation. The real two samples corresponding to two simulations were fabricated to evaluate the model. The simulation and experiment results of forming without compensation are given in section 3.1, whereas results of forming with compensation are given in section 3.2 .

\subsection{Forming process without compensation}

The deformation results of the first simulation are shown in Fig. 13. In this figure, the color of the model represents the deformation (m) on the $\mathrm{x}$ axis. The green dash lines represent the designed position of left or right walls. In the first simulation, they could also be understood as the setting for finishing milling. The gap between the designed lines to the respective left or right wall after deformation was termed the deformation error $\left(\varepsilon_{T}\right)$. Fig. 13 illustrates the deformation error of one point on the left wall of the sample. Using deformation results of simulation, dimensions of sample after deformation were calculated and given in Fig. 15 and Table 4.

The real sample fabricated without compensation is shown in Fig. 14. After fabrication, the sample was measured using coordinate measuring machine $(\mathrm{CMM})$. The measuring path is illustrated in Fig. 14. The diameter of the CMM probe head used was $2 \mathrm{~mm}$, and the measuring step was $0.01 \mathrm{~mm}$. Results of measurements with CMM are shown in Fig. 15

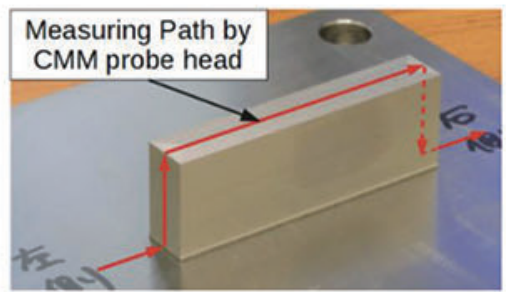

Fig. 14 Measuring left and right walls on the real sample
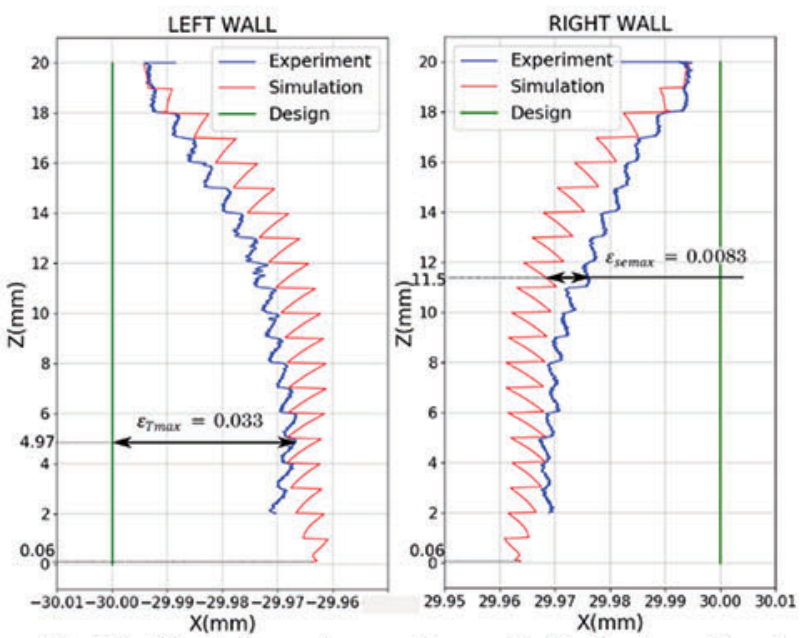

Fig. 15 Simulation and measuring results for forming without compensation

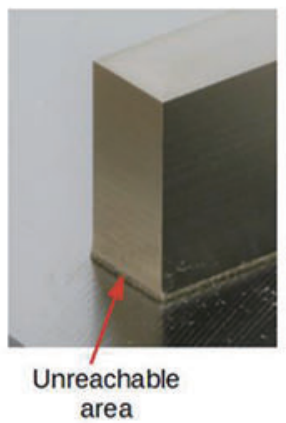

Fig. 16 Area at the bottom of the real part

and Table 4. At the bottom of the real part, there existed a small area that could not be finished by milling (Fig. 16). Therefore, we could not use the measured data at this area for analysis. The measured data from the bottom of the part to $\mathrm{z}=2 \mathrm{~mm}$ were ignored. This problem was mentioned above in section 2.1 .

The maximum deformation error of experiment sample on the left wall was $\varepsilon_{T \max }=0.033 \mathrm{~mm}$ at $\mathrm{z}=4.97 \mathrm{~mm}$ (Fig. 15). In the present study, we used dimensions at the middle of each block to analyze the error between simulation and experiment, and these values are given in Table 4. The error between simulation and experiment at each block was termed simulation error $\left(\varepsilon_{s e}\right)$ and calculated using Eq. (3) below:

$$
\varepsilon_{s e}=\left|D_{s}-D_{e}\right|
$$

where $D_{s}(\mathrm{~mm})$ is the dimension of sample calculated from simulation results, and $D_{e}(\mathrm{~mm})$ is the dimension of the sample measured from experiment. Results of simulation error of each block are given in Table 4. From the simulation errors calculated on both left and right walls, we calculated the mean of $\varepsilon_{s e}$ to be $0.0048 \mathrm{~mm}$, with standard deviation of $0.0019 \mathrm{~mm}$ and the maximum simulation error of $0.0083 \mathrm{~mm}$. 

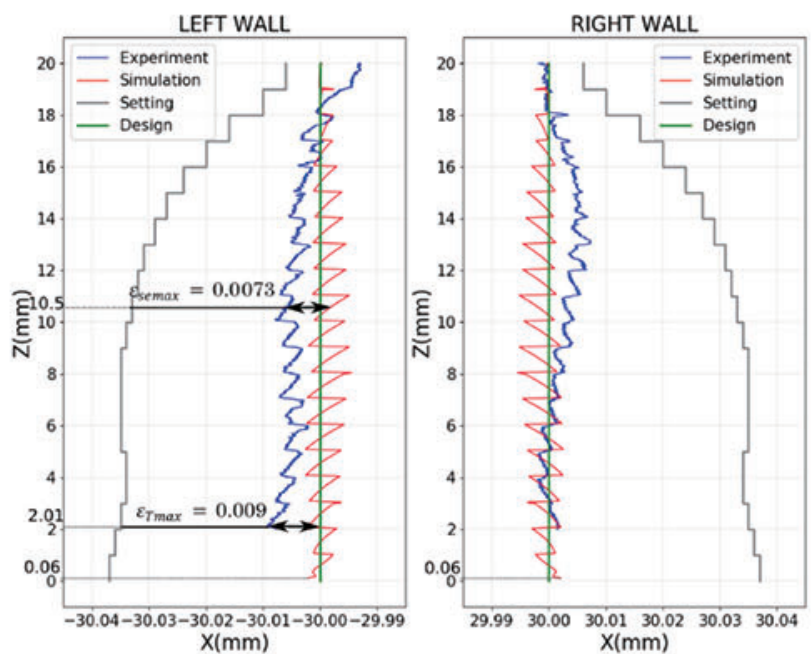

Fig. 17 Simulation and measuring results for forming with compensation

Table 4 Dimensions at the middle of bocks without compensation

\begin{tabular}{rr|r|l|l|l|l}
\hline $\mathbf{Z}(\mathbf{m}$ & \multicolumn{3}{c|}{ Left wall } & \multicolumn{3}{c}{ Right wall } \\
\cline { 2 - 7 } $\mathbf{m})$ & $D_{s}(\mathrm{~mm})$ & $D_{e}(\mathrm{~mm})$ & $\varepsilon_{s e}(\mathrm{~mm})$ & $D_{s}(\mathrm{~mm})$ & $D_{e}(\mathrm{~mm})$ & $\varepsilon_{s e}(\mathrm{~mm})$ \\
\hline 0.5 & -29.9631 & & & 29.9631 & & \\
1.5 & -29.9642 & & & 29.9642 & & \\
2.5 & -29.9649 & -29.9701 & 0.0053 & 29.9649 & 29.9693 & 0.0044 \\
3.5 & -29.9655 & -29.9690 & 0.0035 & 29.9655 & 29.9685 & 0.0029 \\
4.5 & -29.9658 & -29.9684 & 0.0026 & 29.9658 & 29.9684 & 0.0027 \\
5.5 & -29.9655 & -29.9678 & 0.0024 & 29.9655 & 29.9692 & 0.0037 \\
6.5 & -29.9651 & -29.9687 & 0.0035 & 29.9651 & 29.9698 & 0.0047 \\
7.5 & -29.9651 & -29.9692 & 0.0041 & 29.9651 & 29.9700 & 0.0049 \\
8.5 & -29.9655 & -29.9707 & 0.0053 & 29.9655 & 29.9703 & 0.0048 \\
9.5 & -29.9659 & -29.9712 & 0.0053 & 29.9659 & 29.9718 & 0.0059 \\
10.5 & -29.9667 & -29.9719 & 0.0051 & 29.9667 & 29.9727 & 0.0060 \\
11.5 & -29.9678 & -29.9729 & 0.0051 & 29.9678 & 29.9761 & 0.0083 \\
12.5 & -29.9692 & -29.9745 & 0.0053 & 29.9692 & 29.9771 & 0.0078 \\
13.5 & -29.9711 & -29.9770 & 0.0059 & 29.9711 & 29.9790 & 0.0080 \\
14.5 & -29.9733 & -29.9793 & 0.0060 & 29.9733 & 29.9810 & 0.0077 \\
15.5 & -29.9761 & -29.9828 & 0.0066 & 29.9761 & 29.9833 & 0.0072 \\
16.5 & -29.9797 & -29.9856 & 0.0059 & 29.9797 & 29.9852 & 0.0055 \\
17.5 & -29.9840 & -29.9889 & 0.0048 & 29.9840 & 29.9889 & 0.0048 \\
18.5 & -29.9897 & -29.9925 & 0.0028 & 29.9897 & 29.9941 & 0.0043 \\
19.5 & -29.9937 & -29.9930 & 0.0006 & 29.9937 & 29.9941 & 0.0005 \\
\hline & & & & & &
\end{tabular}

\subsection{Forming process with compensation}

The shrinkage during forming process of SHM reduces the final dimensions of the sample as compared with the initial design. The idea of compensation was applied to calculate the deformation owing to shrinkage in the initial designed dimensions of the sample. Subsequently, these values were used to set the finishing milling step. Consequently, after shrinkage, dimensions of the sample should be equal with those of initial design. The compensation setting was defined as the setting value for finishing milling with addition of deformation error to the initial design of the sample. The compensation setting for block $\mathrm{i}^{\text {th }}\left(D_{c i}(\mathrm{~mm})\right)$ was calculated using Eq. (4):

$$
D_{c i}=D_{d}+\left(D_{d}-D_{s i}\right)
$$

Table 5 Compensation setting for finishing milling on the right wall

\begin{tabular}{cc|cc|cc}
\hline Block & $\begin{array}{c}\text { Setting } \\
(\mathbf{m m})\end{array}$ & Block & $\begin{array}{c}\text { Setting } \\
(\mathbf{m m})\end{array}$ & Block & $\begin{array}{c}\text { Setting } \\
(\mathbf{m m})\end{array}$ \\
\hline 1 & 30.037 & 8 & 30.035 & 15 & 30.027 \\
2 & 30.036 & 9 & 30.035 & 16 & 30.024 \\
3 & 30.035 & 10 & 30.034 & 17 & 30.020 \\
4 & 30.034 & 11 & 30.033 & 18 & 30.016 \\
5 & 30.034 & 12 & 30.032 & 19 & 30.010 \\
6 & 30.035 & 13 & 30.031 & 20 & 30.006 \\
7 & 30.035 & 14 & 30.029 & & \\
\hline
\end{tabular}

where $D_{d}$ is the initial setting value for finishing milling based on the designed model $(\mathrm{mm})$ and $D_{s i}(\mathrm{~mm})$ is the dimension after shrinkage at the middle of the $\mathrm{i}^{\text {th }}$ block which is calculated from the first simulation. The compensation setting for the right wall of each block is given in Table 5 . The simulation model used the $\mathrm{z}$-axis as the symmetry axis. Therefore, the compensation setting on the left wall were negative and with absolute value equal to that of the right wall. The setting values are also illustrated in Fig. 16. Consequently, the second simulation and corresponding experiment were conducted. Except the settings for milling were changed with compensation setting given in Table 5 , the other forming conditions were completely similar with those of the first simulation and experiment.

Results of the second simulation and experiment with compensation are shown in Fig. 17 and Table 6. The maximum deformation error of sample in the second experiment, $\varepsilon_{T \max }=0.009 \mathrm{~mm}$ at the left wall and $\mathrm{z}=2.01 \mathrm{~mm}$. The mean of simulation error $\left(\varepsilon_{s e}\right)$ was $0.0037 \mathrm{~mm}$, with standard deviation of $0.0023 \mathrm{~mm}$, and the maximum simulation error of the second simulation, $\varepsilon_{\text {semax }}=0.0073 \mathrm{~mm}$.

\section{Discussion}

The present study discussed two research points. The first included proposing an FEA model to simulate the process of SHM. The effectiveness of the proposed FEA model was evaluated using three criteria: mechanism of deformation, simulation error, and time of calculation. The second point included proposing a compensation technique to reduce the deformation error of SHM. The effectiveness of the proposed compensation technique was evaluated by reducing the deformation error between the first and second experiments. In the present study, we only investigated deformation error on the left and right walls of the part of sample. The reason is that the deformation owing to shrinkage affects directly on these two faces. The error of sample is the combination of error on both left and right wall. The shrinkage affects to the height of sample; however, the deformation error on the height is eliminated when the top of the part is machined.

\subsection{Evaluation of FEA model}

It was predicted that the area close to the shrinking layer would be affected more (Fig. 18). Based on this prediction, the top of a block which is close to the shrinking layer must get more deformed than the bottom of block. This phenomenon was confirmed by both simulation and experiment results, as shown in (Fig. 15). We can see that except the last block, all blocks were smaller at the top and bigger at the bottom. Therefore, we can use this result to confirm our prediction. This is termed the first shrinkage rule. 
Table 6 Dimensions at the middle of bocks with compensation

\begin{tabular}{|c|c|c|c|c|c|c|}
\hline \multirow{2}{*}{$\begin{array}{r}\mathbf{Z}(\mathbf{m} \\
\mathrm{m}) \\
\end{array}$} & \multicolumn{3}{|c|}{ Left wall } & \multicolumn{3}{|c|}{ Right wall } \\
\hline & $D_{s}(\mathrm{~mm})$ & $D_{e}(\mathrm{~mm})$ & $\varepsilon_{s e}(\mathrm{~mm})$ & $D_{s}(\mathrm{~mm})$ & $D_{e}(\mathrm{~mm})$ & $\varepsilon_{s e}(\mathrm{~mm})$ \\
\hline 0.5 & -30.0008 & & & 30.0008 & & \\
\hline 1.5 & -30.0001 & & & 30.0001 & & \\
\hline 2.5 & -30.0005 & -30.0074 & 0.007 & 30.0005 & 30.0012 & 0.0007 \\
\hline 3.5 & -30.0001 & -30.0064 & 0.0064 & 30.0001 & 29.9993 & 0.0007 \\
\hline 4.5 & -30.0004 & -30.0053 & 0.0049 & 30.0004 & 29.9995 & 0.0009 \\
\hline 5.5 & -30.0001 & -30.0049 & 0.0048 & 30.0001 & 29.9998 & 0.0003 \\
\hline 6.5 & -29.9998 & -30.0058 & 0.0061 & 29.9998 & 30.0005 & 0.0007 \\
\hline 7.5 & -29.9997 & -30.0052 & 0.0055 & 29.9997 & 30.0011 & 0.0014 \\
\hline 8.5 & -29.9990 & -30.0050 & 0.0060 & 29.9990 & 30.0023 & 0.0032 \\
\hline 9.5 & -29.9997 & -30.0052 & 0.0055 & 29.9997 & 30.0032 & 0.0036 \\
\hline 10.5 & -29.9987 & -30.0060 & 0.0073 & 29.9987 & 30.0031 & 0.0044 \\
\hline 11.5 & -29.9992 & -30.0058 & 0.0067 & 29.9992 & 30.0045 & 0.0054 \\
\hline 12.5 & -29.9990 & -30.0042 & 0.0052 & 29.9990 & 30.0044 & 0.0054 \\
\hline 13.5 & -29.9993 & -30.0038 & 0.0044 & 29.9993 & 30.0049 & 0.0056 \\
\hline 14.5 & -29.9992 & -30.0042 & 0.0050 & 29.9992 & 30.0053 & 0.0062 \\
\hline 15.5 & -29.9997 & -30.0030 & 0.0033 & 29.9997 & 30.0045 & 0.0048 \\
\hline 16.5 & -30.0000 & -29.9999 & 0.0000 & 30.0000 & 30.0031 & 0.0032 \\
\hline 17.5 & -29.9992 & -30.0015 & 0.0023 & 29.9992 & 30.0008 & 0.0016 \\
\hline 18.5 & -29.9999 & -29.9992 & 0.0007 & 29.9999 & 29.9996 & 0.0003 \\
\hline 19.5 & -30.0001 & -29.9941 & 0.0060 & 30.0001 & 29.9992 & 0.0009 \\
\hline
\end{tabular}

After the first shrinkage rule was confirmed, another prediction was that the distance between a specific block and the top of fabricating sample increased after forming a new block. Based on the first shrinkage rule, if another block was continuously formed, the shrinkage effect on this specific block must be reduced. It was predicted that after a specific number of blocks, the shrinkage effect on that block was not significant anymore. This prediction was defined as the second shrinkage rule, which explained why deformations from block 2 to block 8 were nearly similar and did not reduce continuously from block 8 to block 20 (Fig. 15). Therefore, we can conclude that after approximate 12 blocks, the shrinkage effect on a block was ineffective.

SHM process using step machining process explained above is a specific process. Therefore, thermal deformation error evolution in SHM also follows a specific process. As mentioned above, after step 2, block 2 was formed and rough machined, and block 1 was finishing machined. In the present study, we assumed that the milling error was not significant; thus, error in block 1 at this step was assumed to be zero (Fig. 19 (a)). In the next step, new layers of block 3 were added. Consequently, block 3 was rough machined and block 2 was finished machined. Shrinkage during the cooling process of formation of block 3 created deformation in both blocks 1 and 2 . However, while the deformation in block 2 was eliminated by milling, that in on block $1\left(\varepsilon_{T 1}\right)$ remained (Fig. 19 (b)). Similarly, after forming block 4 , deformation in block $2\left(\varepsilon_{T 2}\right)$ appeared and that in block 1 continuously increased, as illustrated in Fig. 19 (c). This process continued until the last block was formed. This process described how deformation error was created; it is named the third shrinkage rule. This rule explains why deformation of a block is greater than that of the above block. This rule is clearly shown from block 8 to block 20 of the first simulation and experiment (Fig. 15).

As mentioned in section 2.1, unclamping was considered as step 22 in simulation owing to the significant deformation that it could create on the

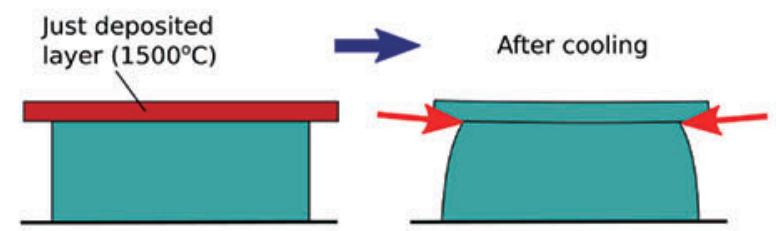

Fig. 18 Mechanism of shrinkage (the first shrinkage rule)

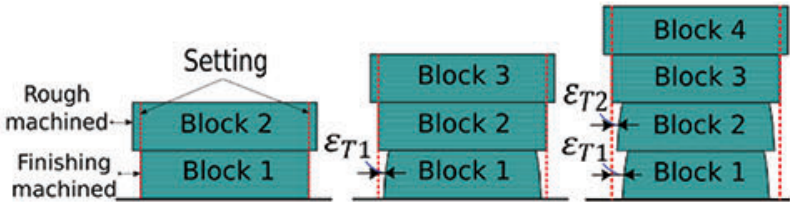

(a) After forming

(b) After formin block 3

(c) After forming block 2 block 4

Fig. 19 Thermal deformation error evolution (the third shrinkage rule)

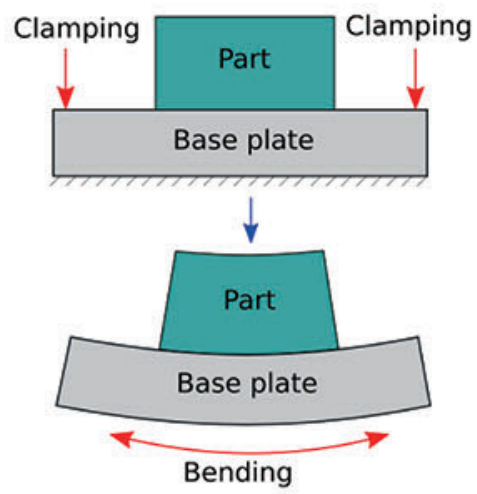

Fig. 20 Deformation owing to unclamping

sample. As our initial prediction, unclamping would create the bending on the sample as shown in Fig. 20 (Fig. 20 only considered unclamping effect). Effect of shrinkage created the compressive stress at the top the base plate; in contrast, the bottom of base plate was affected by tensile stress. After released the base plate from the workstation, the residual stress made it bend as shown in Fig. 20. The part of sample was affected by the bending of the base plate and deformed similarly. Base on this mechanism, unclamping would create as more deformation error at the top of the part than at the bottom. The error that was created owing to unclamping of the base plate was termed of unclamping error $\left(e_{u c}\right), e_{u c}$ was calculated by Eq. (5):

$$
e_{u c}=D_{s}-D_{s b u}
$$

where $D_{s}$ is the final dimensions of sample (dimension after unclamping) which is defined in section 3.1, and $D_{s b u}$ is the dimensions of the sample before unclamping. From the result of the first simulation, unclamping errors on the left wall, at the middle of all blocks were calculated and are shown in Table 7. In general, unclamping errors reduces from the top to the bottom of the part. The unclamping error at block 20 was $0.0091 \mathrm{~mm}$ which is the maximum one. The unclamping errors of the next blocks continuously reduce to $0.003 \mathrm{~mm}$ at block 4 . However, from block 4 to block 1 , the unclamping error lightly increased to $0.0043 \mathrm{~mm}$. The simulation results were similar with the initial prediction except the unclamping errors at the three last blocks which were larger than expectation. The reason of this unexpected error may be owing to the fact 


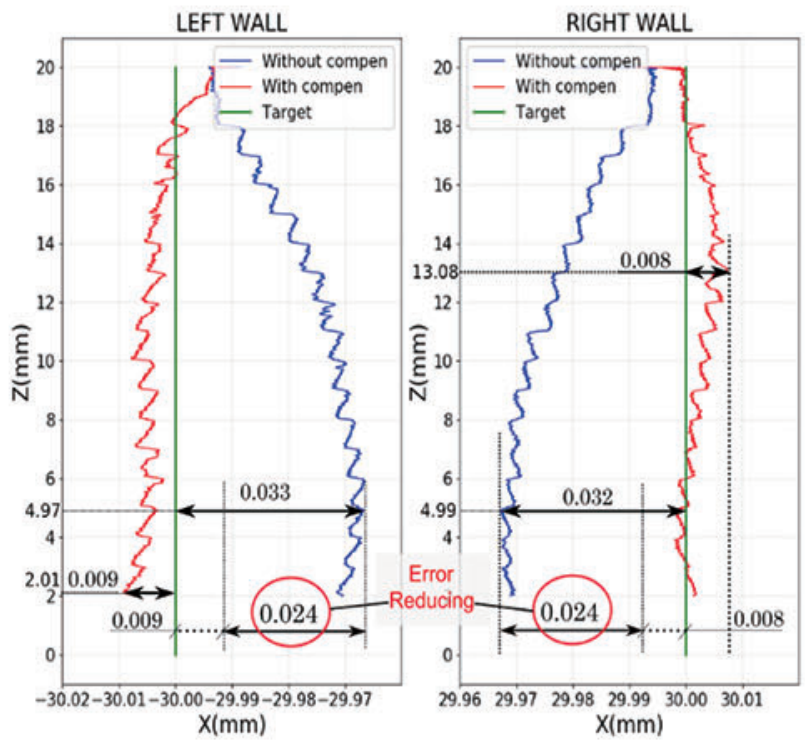

Fig. 21 Efficiency of compensation in reducing deformation

Table 7 Deformation owing to unclamping on the left wall

\begin{tabular}{cc|cc|cc}
\hline $\mathbf{Z}(\mathbf{m m})$ & $\begin{array}{c}\left(e_{u c}\right) \\
(\mathbf{m m})\end{array}$ & $\mathbf{Z}(\mathbf{m m})$ & $\begin{array}{c}\left(e_{u c}\right) \\
(\mathbf{m m})\end{array}$ & $\mathbf{Z}(\mathbf{m m})$ & $\begin{array}{c}\left(e_{u c}\right) \\
(\mathbf{m m})\end{array}$ \\
\hline 0.5 & 0.0043 & 7.5 & 0.0039 & 14.5 & 0.0060 \\
1.5 & 0.0035 & 8.5 & 0.0038 & 15.5 & 0.0066 \\
2.5 & 0.0033 & 9.5 & 0.0040 & 16.5 & 0.0072 \\
3.5 & 0.0030 & 10.5 & 0.0043 & 17.5 & 0.0078 \\
4.5 & 0.0031 & 11.5 & 0.0047 & 18.5 & 0.0084 \\
5.5 & 0.0032 & 12.5 & 0.0051 & 19.5 & 0.0091 \\
6.5 & 0.0033 & 13.5 & 0.0055 & & \\
\hline
\end{tabular}

that block 1 is the area where the base plate directly transferred the bending effect to the part. Moreover, dimension of sample suddenly reduced from $125 \mathrm{~mm}$ (at the top of the base plate) to $60 \mathrm{~mm}$ (at the bottom of the part). We believe that the reasons mentioned above caused a special enhance effect made the unclamping error at the bottom of the part increased. The results of the simulation were not enough to confirm this phenomenon; moreover, we could not take experiment result at the bottom of the part as mentioned in section 3.1 and Fig. 16. Therefore, in the present research, we ignored the effect of unclamping error enhancing at the bottom of part. The initial prediction about unclamping effect on deformation error is confirmed by the simulation results from block 4 to block 20 .

The simulation results helped gain a better understanding of the mechanism of deformation in SHM. Since the result of experiment sample were similar with those of simulation, we confirmed that the mechanism of shrinkage in our FEA model was appropriate.

The accuracy of the model was evaluated using simulation error $\left(\varepsilon_{s e}\right)$ given in Table 4 in the "Result" section. The average simulation error of the model was estimated to be $0.0048 \mathrm{~mm}$, calculated using the values of $\varepsilon_{s e}$ at the middle of blocks (Table 4), with a standard deviation of 0.0019 $\mathrm{mm}$. The maximum simulation error was $0.0083 \mathrm{~mm}$ at block 12 of the right wall (Fig. 15 and Table 4). While the initial accuracy requirement of the model was $0.01 \mathrm{~mm}$, this result satisfied the requirement. Time of simulation is another specific requirement of the FEA model. Shorter the simulation time, better the model. In this simulation, time for calculation was approximately $48 \mathrm{~h}$. In case of real application, it properly took considerably long to calculate compensation data for a sample. However, this is acceptable in experimental research.

\subsection{Efficiency of compensation technique}

To reduce the thermal deformation error, compensation technique was applied. The target and technique of compensation are explained in the "Result" section. Results of the experiment without compensation are depicted in Fig. 15 that show the maximum deformation error to be 0.033 $\mathrm{mm}$ at block 5 of the left wall. The experiment with compensation in Fig. 17 shows the maximum deformation error as $0.009 \mathrm{~mm}$ at block 3 of the left wall. The deformation error was reduced to $0.024 \mathrm{~mm}$ on the left side of sample, and also to $0.024 \mathrm{~mm}$ on the right side of the sample. Fig. 21 shows in detail the error reducing between the results of the first and the second experiment. In the present research, attaining an error less than 0.01 $\mathrm{mm}$ was considered as a significant improvement.

In Fig. 21, we see that the error without compensation is symmetric while that of with compensation is not. This phenomenon is explained as the following. The error without using compensation contained not only thermal deformation error but also other errors such as milling error, measuring error. The thermal deformation error is more significant than the others. Moreover, the thermal deformation error is symmetric, therefore, the results without using compensation look symmetric. In contrast, after using compensation, the thermal deformation error is almost eliminated, while the remaining errors are randomly and asymmetric. Therefore, the results with compensation are asymmetric.

Analysis for simulation error $\left(\varepsilon_{s e}\right)$ of forming with compensation is described in section 3 . The simulation errors $\left(\varepsilon_{s e}\right)$ at the middle of each block are given in Table 6. Using data in Table 6, the mean of $\varepsilon_{s e}$ was determined to be $0.0037 \mathrm{~mm}$, with standard deviation $0.0023 \mathrm{~mm}$, and the $\varepsilon_{\text {semax }}$ was $0.0073 \mathrm{~mm}$ (Fig. 17). The analysis result of $\varepsilon_{s e}$ in case of with compensation is nearly similar with the results in case of without compensation. It proved that the FEA model worked efficiently in both cases, and again, confirmed the effectiveness of the proposed FEA model.

\subsection{Potential and limitations of model for future research}

The most importance target of this study was to develop an FEA model to replace the approach used in studying thermal deformation error in SHM. With the FEA model, the effect of several processing parameters, such as width, height of sample, and block thickness, on deformation error could be investigated. The second target of the study was to find a solution to the problem of thermal deformation error in SHM. Results of our second experiment proved that thermal deformation error in SHM could be eliminated by compensation.

As mentioned above, the time of simulation is a fundamental limitation of FEA model. Another limitation of this model is that 2D simulation could not investigate the effectiveness of the depth of sample. These problems will be considered in the future studies.

\section{Conclusions}

This study achieved two important targets: building an efficient FEA model to investigate thermal deformation error in SHM and finding a potential technique to reduce this error. The results of the first simulation helped understand the shrinkage mechanism with three shrinkage rules. The second simulation proved that the compensation technique could significantly reduce thermal deformation error of SHM. The results of two corresponding experiments also confirmed the results of simulations. Although the present study had certain limitations, we believe that the FEA 
model could be used to investigate several parameters of SHM in the future studies.

\section{References}

1) H. Bikas, P. Stavropoulos, and G. Chryssolouris, "Additive manufacturing methods and modeling approaches: A critical review," Int. J. Adv. Manuf. Technol., vol. 83, no. 1-4, pp. 389-405, 2016.

2) W. Du, Q. Bai, and B. Zhang, "A Novel Method for Additive/Subtractive Hybrid Manufacturing of Metallic Parts," Procedia Manuf., vol. 5, pp. 1018-1030, 2016.

3) M. Behandish, S. Nelaturi, and J. De Kleer, "ComputerAided Design Automated process planning for hybrid manufacturing," Comput. Des., vol. 102, pp. 115-127, 2018.

4) W. Du, Q. Bai, and B. Zhang, "Machining characteristics of $18 \mathrm{Ni}-300$ steel in additive/subtractive hybrid manufacturing," Int. J. Adv. Manuf. Technol., vol. 95, no. 5-8, pp. 2509-2519, 2018.

5) Y. A. Song, S. Park, D. Choi, and H. Jee, "3D welding and milling: Part I-a direct approach for freeform fabrication of metallic prototypes," Int. J. Mach. Tools Manuf., vol. 45, no. 9, pp. 1057-1062, 2005.

6) J. Fessler, R. Merz, A. Nickel, and F.B. Prinz, "Laser deposition of metals for shape deposition manufacturing," Proceedings of the Solid Freeform Fabrication Symposium, pp. 117-124, 1996.

7) Z. peng Ye, Z. jing Zhang, X. Jin, M. Z. Xiao, and J. zhou $\mathrm{Su}$, "Study of hybrid additive manufacturing based on pulse laser wire depositing and milling," Int. J. Adv. Manuf. Technol., vol. 88, no. 5-8, pp. 2237-2248, 2017.

8) https://www.sodick.com/products/metal-3dprinting/opm2501 (Sodick, Metal 3D Printer OPM250L) (Access: July 19, 2019).

9) H. Y. Wan, Z. J. Zhou, C. P. Li, G. F. Chen, and G. P. Zhang, "Effect of scanning strategy on mechanical properties of selective laser melted Inconel 718," Mater. Sci. Eng. A, vol. 753, no. December 2018, pp. 42-48, 2019.

10) J. P. Kruth, L. Froyen, J. Van Vaerenbergh, P. Mercelis, M. Rombouts, and B. Lauwers, "Selective laser melting of ironbased powder," J. Mater. Process. Technol., vol. 149, no. 13, pp. 616-622, 2004.

11) J. Delgado, J. Ciurana, and C. A. Rodríguez, "Influence of process parameters on part quality and mechanical properties for DMLS and SLM with iron-based materials," Int. J. Adv. Manuf. Technol., vol. 60, no. 5-8, pp. 601-610, 2012.

12) H. Shipley et al., "Optimisation of process parameters to address fundamental challenges during selective laser melting of Ti-6Al-4V: A review," Int. J. Mach. Tools Manuf., vol. 128, no. January, pp. 1-20, 2018.

13) T. Simson, A. Emmel, A. Dwars, and J. Böhm, "Residual stress measurements on AISI 316L samples manufactured by selective laser melting," Addit. Manuf., vol. 17, pp. 183-189, 2017.

14) P. Mercelis and J. P. Kruth, "Residual stresses in selective laser sintering and selective laser melting," Rapid Prototyp.
J., vol. 12, no. 5, pp. 254-265, 2006.

15) J. Robinson, I. Ashton, P. Fox, E. Jones, and C. Sutcliffe, "Determination of the effect of scan strategy on residual stress in laser powder bed fusion additive manufacturing," Addit. Manuf., vol. 23, no. February, pp. 13-24, 2018.

16) C. Li, J. F. Liu, X. Y. Fang, and Y. B. Guo, "Efficient predictive model of part distortion and residual stress in selective laser melting," Addit. Manuf., vol. 17, pp. 157-168, 2017.

17) L. Parry, I. A. Ashcroft, and R. D. Wildman, "Understanding the effect of laser scan strategy on residual stress in selective laser melting through thermo-mechanical simulation," Addit. Manuf., vol. 12, pp. 1-15, 2016.

18) J. Wu, L. Wang, and X. An, "Numerical analysis of residual stress evolution of AlSi10Mg manufactured by selective laser melting," Optik (Stuttg)., vol. 137, pp. 65-78, 2017.

19) J. P. Kruth, J. Deckers, E. Yasa, and R. Wauthlé, "Assessing and comparing influencing factors of residual stresses in selective laser melting using a novel analysis method," Proc. Inst. Mech. Eng. Part B J. Eng. Manuf., vol. 226, no. 6, pp. 980-991, 2012.

20) M. Shiomi, K. Osakada, K. Nakamura, T. Yamashita, and F. Abe, "Residual stress within metallic model made by selective laser melting process," CIRP Ann. - Manuf. Technol., vol. 53, no. 1, pp. 195-198, 2004.

21) A. Foroozmehr, M. Badrossamay, E. Foroozmehr, and S. Golabi, "Finite Element Simulation of Selective Laser Melting process considering Optical Penetration Depth of laser in powder bed," Mater. Des., vol. 89, pp. 255-263, 2016.

22) X. C. Wang, T. Laoui, J. Bonse, J. P. Kruth, B. Lauwers, and L. Froyen, "Direct selective laser sintering of hard metal powders: Experimental study and simulation," Int. J. Adv. Manuf. Technol., vol. 19, no. 5, pp. 351-357, 2002.

23) M. Chiumenti et al., "Numerical modelling and experimental validation in Selective Laser Melting," Addit. Manuf., vol. 18, pp. 171-185, 2017.

24) https://www.tokkin.com/materials/special_steel/carbon_stee 1 (Tokkin, Carbon Steels (S15C, S45C, S50C, S55C, \& S60C)) (Access: July 19, 2019).

25) https://www.tokkin.com/materials/high_parformance/mas1 (Tokkin, Maraging Steels (MAS-1)) (Access: July 19, 2019).

26) https://www.j-platpat.inpit.go.jp/c1800/PU/JP3687672/5BFF8032FAF1D1FC141F284D26A5CA81777A C0506B4AA07932FBF5F9E5989814/15/ja (Panasonic, Finishing Methods for Powder Sinking Parts) (Access: July 19, 2019).

27) http://resources.renishaw.com/en/download/data-sheetmaraging-steel-m300-for-200-w-powder-for-additivemanufacturing--96325 (Maraging steel M300 powder for additive manufacturing) (Access: September 9, 2019).

28) https://www.code-aster.org/V2/doc/default/en/man_r $\mathrm{r} / \mathrm{r} 5 / \mathrm{r} 5.0$ 3.02.pdf (Integration of the relations of behavior elastoplastic of Von Mises). 